\title{
Dynamics of the Tumor-Obesity with Time Delay Effect
}

\author{
S. Udomchalermpat ${ }^{1}$, S. Koonprasert ${ }^{2}$ and E. Kunnawuttipreechachan ${ }^{3}$ \\ ${ }^{1}$ Lecturer, Mathematics, School of General Education, Kasem Bundit University, Bangkok 10250, \\ Thailand \\ ${ }^{2}$ Associate Professor, Department of Mathematics, Faculty of Applied Science, \\ King Mongkut's University of Technology North Bangkok, Bangkok, 10800, Thailand. \\ ${ }^{3}$ Lecturer, Department of Mathematics, Faculty of Applied Science, \\ King Mongkut's University of Technology North Bangkok, Bangkok, 10800, Thailand.
}

ORCIDs: 0000-0003-2165-2393 (Udomchalermpat), 0000-0001-5236-033X (Koonprasert)

\begin{abstract}
In this paper, the obesity and tumor model has been analyzed. We aim to show that all solutions of the model are bounded and find all equilibria of the models. We also investigate the conditions for the existence of positive equilibria for the models. Next, the local and global stability of the positive equilibrium is determined by the linearization and Lyapunov methods. Moreover, the model with time-delay is also exhibited for the existence of a Hopf bifurcation. Finally, we illustrate the numerical results using some advantages of mathematical software to support the analytic results and show the effect of some parameters for tumor growth.
\end{abstract}

Keywords: Obesity; Tumor model; Time delay; Stability; Hopf bifurcation.

\section{INTRODUCTION}

A tumor is formed in body due to abnormal cellular growth, and it becomes cancer when the tumor is malignant. It is one of the most serious world health problems [1]. There are many common causes of tumor, such as, smoking and tobacco, diet and physical activity, sun and other types of radiation, viruses and other infections. Recently, many previous works [2-6] show that obesity is a risk factor for many serious diseases such as type-II-diabetes, hypertension, hearth problem, including tumor and cancer. Therefore, the relationship between obesity and tumor growth is an interested topic for many researchers.

Observing several experimental studies between cancer and obesity, [7,8,21] the International Agency for Research on Cancer (IARC) has reported linkages between cancer and obesity in cases of colorectal cancer, breast cancer in postmenopausal women, endometrium cancer, renal cancer, and oesophagus cancer [9]. In previous work [8-10], it has been found that obesity and excess weight are two major health problems in countries around the world. These problems are mostly caused by a sedentary lifestyle and excess eating. Obesity occurs when excessive amounts of fat cells are stored in the body. It is well known that the fat cell population increases rapidly during childhood. In adulthood, the fat cell population remains almost constant or increases slowly unless there is a dramatic weight gain or loss [11]. In 2016, the World Health Organization (WHO) reported that approximately 2.3 billion adults in the world were overweight and more than 700 million people were obese. Obesity can cause other serious diseases which could be fatal, for example hypertension, heart problems, cancer, tumors, etc.

In theoretical cancer researches, mathematical modeling is one of the more successful methodologies by applying experimental data to create mathematical equations with describing tumor growth. In 2013, Okwan-Duodu et al. [12] studied simulation models to assess the effect of obesity on mortality of cancer patients. In 2016, Ku-Carrillo et al. assumed that the obesity of an individual is directly proportional to the carrying capacity of their body to store fat which means that the obesity degree of an individual can carrying in the organism which tumor can be occurred, so obesity can carry in tumor.

In 2003, Villasana et al. [13] developed the logistic growth function with time delay for studying the effect of drug to the tumor cells by tumor-growth model. In 2014, Rihan et al. [14] had shown that a time delay between the interactions of the immune cells and the tumor cells and the growth rate of the immune cells are important for developing a suitable response after recognizing the tumor cells. In this work, we extend the model interaction between tumor cells and obesity represented by the positive nonlinear growth term for the immune cells $\frac{\rho I(t-\tau) T(t-\tau)}{\alpha+T(t-\tau)}$ and the competition between immune cells and tumor cells $c_{1} I(t-\tau) T(t-\tau)$ with a time delay $(\tau)$. Hence, we generalized the model for the interaction between tumor and obesity as

$$
\begin{aligned}
I \dot{(t)} & =s+\frac{\rho I(t-\tau) T(t-\tau)}{\alpha+T(t-\tau)}-c_{1} I(t-\tau) T(t-\tau)-d_{1} I(t), \\
\dot{T(t)} & =r_{1} T(t)\left(1-b_{1} T(t)\right)-c_{2} I(t) T(t)-c_{3} T(t) N(t)+c_{5} T(t) F(t),(1) \\
N(t) & =r_{2} N(t)\left(1-b_{2} N(t)\right)-c_{4} T(t) N(t), \\
F(t) & =r_{3} F(t)\left(1-b_{3} F(t)\right),
\end{aligned}
$$

where $I(t)$ is the density of immune cells at time $t, T(t)$ is the density of cancer - tumor cells at time $t, N(t)$ is the density of host cells at time $t, F(t)$ represents the density of fat cells at time $t$ and $\tau \geq 0$. The parameters of model (1) are in the Table 1 as follow 
International Journal of Engineering Research and Technology. ISSN 0974-3154, Volume 13, Number 8 (2020), pp. 1854-1865

(C) International Research Publication House. http://www.irphouse.com

Table 1. The value of parameters for the tumor-obesity model

\begin{tabular}{|c|c|c|}
\hline Parameters & Meaning & Unit \\
\hline$s$ & A constant rate of migration of immune cells into the tumor & Density. $m L^{-1} \cdot d a y^{-1}$ \\
\hline$\rho$ & A positive constant & $d a y^{-1}$ \\
\hline$\alpha$ & A positive constant & Density. $m L$ \\
\hline$d_{1}$ & The natural death rate of the immune cells & $d a y^{-1}$ \\
\hline$r_{1}$ & The growth rate for the cancer cells & $d a y^{-1}$ \\
\hline$r_{2}$ & The growth rate for the normal cells & $d a y^{-1}$ \\
\hline$r_{3}$ & The growth rate for the density of fat cells & $d a y^{-1}$ \\
\hline$b_{1}$ & The inverse of the carrying capacity for the tumor cells & Density. $m L$ \\
\hline$b_{2}$ & The inverse of the carrying capacity for the normal cells & Density. $m L$ \\
\hline$b_{3}$ & The inverse of the carrying capacity for the density of fat & Density. $m L$ \\
\hline$c_{1}$ & The competition coefficients between immune cells and tumor cells & Density $\cdot m L^{-1} \cdot d a y^{-1}$ \\
\hline$c_{2}$ & The competition coefficients between tumor cells and immune cells & Density $\cdot m L^{-1} \cdot$ day $^{-1}$ \\
\hline$c_{3}$ & The competition coefficients between tumor cells and normal cells & Density. $m L^{-1} \cdot d a y^{-1}$ \\
\hline$c_{4}$ & The competition coefficients between normal cells and tumor cells & Density. $m L^{-1} \cdot d a y^{-1}$ \\
\hline$c_{5}$ & The competition coefficients between tumor cells and fat cells & Density. $m L^{-1} \cdot d a y^{-1}$ \\
\hline
\end{tabular}

Note that all variables are assumed to be non-negative and all parameters are assumed to be positive.

\section{BOUNDEDNESS OF SOLUTIONS}

We now prove that all solutions of the model are bounded using the LCIS method [15] to find the bounds of a domain containing all compact invariant sets of model (1) with $\tau=0$ for positive values of model parameters.

First, we will find ultimate densities of fat cells by taking the function $h_{1}=F$ with $F>0$. The derivative was computed as

$$
L_{f} h_{1}=r_{3} F\left(1-b_{3} F\right)
$$

By solving this differential equation, then the maximum density of fat cells is

$$
K_{1}\left(h_{1}\right)=\left\{F \leq F_{\max }:=\frac{1}{b_{3}}\right\} .
$$

Hence, the density of fat cells is bounded as

$$
K\left(h_{1}\right)=\left\{0 \leq F(t) \leq \frac{1}{b_{3}}\right\} .
$$

Applying the localizing function $h_{2}=T$ with $T>0$. From (1), the Lie derivative of $h_{2}$ can be defined as follows

$$
L_{f} h_{2}=r_{1} T\left(1-b_{1} T\right)-c_{2} I T-c_{3} T N+c_{5} T F .
$$

By solving this differential equation, then the maximum density of tumor cells without taking the immune cells and the normal cells is

$$
K_{1}\left(h_{2}\right)=\left\{T \leq \frac{1}{b_{3}}+\frac{c_{5}}{r_{1} b_{1} b_{3}}\right\} .
$$

Taking the function $h_{3}=N$ with $N>0$, then the derivative was computed as

$$
L_{f} h_{3}=r_{2} N\left(1-b_{2} N\right)-c_{4} T N \text {. }
$$

By solving this differential equation, we can get the set

$$
S\left(h_{3}\right) \cap\{N>0\}=\left\{N=\frac{1}{b_{2}}-\frac{c_{4} T}{r_{2} b_{2}}\right\} .
$$

Hence, the ultimate density of normal cells is denoted by the set

$$
K\left(h_{3}\right)=\left\{0 \leq N(t) \leq \frac{1}{b_{2}}\right\} .
$$

Next, upper and lower bounds for the density of immune cells can be determined by taking the function $h_{4}=I$. We computed its Lie derivative as follows

$$
L_{f} h_{4}=s+\frac{\rho I T}{\alpha+T}-c_{1} I T-d_{1} I .
$$

Now, in order to derive the lower bound, we take all negative terms to the left side of the equation and by neglecting the rational term in the right side and applying the Iterative Theorem we get

$$
S\left(h_{4}\right) \cap K_{1}\left(h_{2}\right) \subset\left\{\left(c_{1}\left(\frac{1}{b_{3}}+\frac{c_{5}}{r_{1} b_{1} b_{3}}\right)+d_{1}\right) I \geq s\right\} .
$$


International Journal of Engineering Research and Technology. ISSN 0974-3154, Volume 13, Number 8 (2020), pp. 1854-1865

(C) International Research Publication House. http://www.irphouse.com

Hence, we establish that the lower bound for the density of immune cells is given by

$$
K_{1}\left(h_{4}\right)=\left\{I \geq I_{\text {min }}:=\frac{s r_{1} b_{1} b_{3}}{c_{1} r_{1} b_{3}+c_{1} c_{5}+r_{1} b_{1} b_{3} d_{1}}\right\} .
$$

For upper bound, by neglecting negative terms in the right side and applying the Iterative Theorem, we obtain

$$
S\left(h_{4}\right) \subset\left\{\left(d_{1}-\rho\right) I \leq s\right\} .
$$

If the condition holds

$$
d_{1}-\rho>0
$$

Thus, we can conclude that the upper bound for the density of immune cells is given by

$$
K_{2}\left(h_{4}\right)=\left\{I \leq I_{\max }:=\frac{s}{d_{1}-\rho}\right\} .
$$

Hence, the ultimate density of immune cells is defined as

$$
K\left(h_{4}\right)=\left\{\frac{s r_{1} b_{1} b_{3}}{c_{1} r_{1} b_{3}+c_{1} c_{5}+r_{1} b_{1} b_{3} d_{1}} \leq I(t) \leq \frac{s}{d_{1}-\rho}\right\} .
$$

Finally, we can define the upper bound for the action of the density of immune cells and density of normal cells on density of tumor cells. We assume that

$$
\begin{aligned}
r_{1}-c_{2} I_{\text {min }}-c_{3} N_{\text {min }} & >0, \\
r_{1}-c_{2} I_{\text {min }} & >0 .
\end{aligned}
$$

From $S\left(h_{2}\right) \cap\{T>0\}$, we apply the Iterative Theorem as

$S\left(h_{2}\right) \cap\{T>0\} \cap K\left(h_{3}\right) \cap K\left(h_{4}\right) \subset\left\{T \leq T_{\max }:=\frac{1}{b_{1}}+\frac{c_{5}}{r_{1} b_{1} b_{3}}-\frac{c_{2} I_{\min }+c_{3} N_{\min }}{r_{1} b_{1}}\right\}$.

We can define the boundedness of density of tumor cells as

$$
K\left(h_{2}\right)=\left\{0 \leq T \leq \frac{1}{b_{1}}+\frac{c_{5}}{r_{1} b_{1} b_{3}}-\frac{c_{2} I_{\min }+c_{3} N_{\min }}{r_{1} b_{1}}\right\} .
$$

From the results of (2), (3), (5) and (7), we can conclude the theorem as follow

Theorem 1 All compact invariant sets of the model (1) with $\tau=0$ are bounded with conditions (4) and (6) in the positively invariant domain $W:=R_{+, 0}^{4}$ are located inside the bounded domain $K:=W \cap K\left(h_{1}\right) \cap K\left(h_{2}\right) \cap K\left(h_{3}\right) \cap K\left(h_{4}\right)$, where

$$
\begin{gathered}
I_{\text {min }} \leq I(t) \leq \frac{s}{d_{1}-\rho} ; \quad d_{1}-\rho>0, \\
0 \leq T(t) \leq \frac{1}{b_{1}}+\frac{c_{5}}{r_{1} b_{1} b_{3}}-\frac{c_{2} I_{\min }}{r_{1} b_{1}} ; \quad r_{1}-c_{2} I_{\text {min }}>0, \\
0 \leq N(t) \leq \frac{1}{b_{2}}, \quad 0 \leq F(t) \leq \frac{1}{b_{3}},
\end{gathered}
$$

and $I_{\min }=\left(s r_{1} b_{1} b_{3}\right) /\left(c_{1} r_{1} b_{3}+c_{1} c_{5}+r_{1} b_{1} b_{3} d_{1}\right)$.
In case $\tau>0$, we also show that $I(t)$ of the first equation of (1) is uniformly bounded by using the generalized Gronwall Lemma [16]. From the first equation of model (1), we obtain

$$
I(t)=e^{-d_{1} t}\left(I(0)+\int_{0}^{t}\left(s+\frac{\rho I(u-\tau) T(u-\tau)}{\alpha+T(u-\tau)}-c_{1} I(u-\tau) T(u-\tau)\right) e^{d_{1} u} d u\right) .
$$

Since $T /(\alpha+T)<1$ and $e^{-d_{1} t} \in(0,1]$, we get

$$
\begin{aligned}
I(t) & \leq I(0)+\frac{s}{d_{1}} e^{d_{1} t}+\int_{0}^{t} \rho I(u-\tau) e^{d_{1} u} d u, \\
& <I(0)+\frac{s}{d_{1}} e^{d_{1} t}+\int_{0}^{t}\left(\rho e^{d_{1}(u+\tau)}\left(I(0)+\frac{s}{d_{1}} e^{d_{1} u}\right) e^{\int_{u}^{t} \rho e^{d_{1}(\xi+\tau)} d \xi}\right) d u(9) \\
& =M_{1} .
\end{aligned}
$$

The generalized Gronwall Lemma gives $I(t)<M_{1}$ where $M_{1}$ is uniformly bounded, then $I(t)$ is uniformly bounded.

\section{ANALYSIS OF EQUILIBRIA}

In this section, we derive conditions for the existence of equilibrium populations $\left(I^{*}, T^{*}, N^{*}, F^{*}\right)$. From the last equation of (1), it is obvious that $F^{*}=0$ or $1 / b_{3}$. If $F^{*}=0$, i.e., no fat inside the tumor, then the equilibrium points are

$$
E_{1}=\left(\frac{s}{d_{1}}, 0,0,0\right), E_{2}=\left(\frac{s}{d_{1}}, 0, \frac{1}{b_{2}}, 0\right), E_{3}=\left(I^{*}, T^{*}, 0,0\right)
$$

and $E_{4}=\left(I^{*}, T^{*}, N^{*}, 0\right)$,

where

$$
T^{*}=\frac{r_{2} c_{3}-r_{1} r_{2} b_{2}+r_{2} b_{2} c_{2} c_{4} I^{*}}{c_{3} c_{4}-r_{1} r_{2} b_{1} b_{2}} \text { and } N^{*}=\frac{r_{1} c_{4}-r_{1} r_{2} b_{1}+c_{2} c_{4} I^{*}}{c_{3} c_{4}-r_{1} r_{2} b_{1} b_{2}} \text {. }
$$

Therefore, $T^{*}$ and $N^{*}$ are positive real number if the following condition is satisfied when $c_{3} c_{4}>r_{1} r_{2} b_{1} b_{2}$ and $I^{*}>\max \left\{\frac{r_{1} b_{2}-c_{3}}{b_{2} c_{2} c_{4}}, \frac{r_{1} r_{2} b_{1}-r_{1} c_{4}}{c_{2} c_{4}}\right\}$. The value of $I^{*}$ satisfies the cubic equation

$$
I^{* 3}+a_{2} I^{* 2}+a_{1} I^{*}+a_{0}=0,
$$

where

$$
\begin{aligned}
a_{2}= & \frac{-1}{r_{2} b_{2} c_{1} c_{2}}\left(\left(c_{3} c_{4}-r_{1} r_{2} b_{1} b_{2}\right)\left(\alpha c_{1}+d_{1}+\rho\right)+2 c_{1} r_{2}\left(r_{1} b_{2}+c_{3}\right)\right), \\
a_{1}= & \frac{1}{r_{2}^{2} b_{2}^{2} c_{1} c_{2}^{2}}\left(\alpha d_{1}\left(c_{3} c_{4}-r_{1} r_{2} b_{1} b_{2}\right)^{2}+c_{1} r_{2}^{2}\left(r_{1}^{2} b_{2}^{2}-2 r_{1} b_{2} c_{3}+c_{3}^{2}\right)\right. \\
& \left.+r_{2}\left(c_{3} c_{4}-r_{1} r_{2} b_{1} b_{2}\right)\left(s b_{2} c_{2}+\alpha c_{1} r_{1} b_{2}-\alpha c_{1} c_{3}+d_{1} r_{1} b_{2}-d_{1} c_{3}-\rho r_{1} b_{2}+\rho c_{3}\right)\right), \\
a_{0}= & \frac{s\left(c_{3} c_{4}-r_{1} r_{2} b_{1} b_{2}\right)}{r_{2}^{2} b_{2}^{2} c_{1} c_{2}^{2}}\left(c_{3}\left(r_{2}+\alpha c_{4}\right)-r_{1} r_{2} b_{2}\left(1+\alpha b_{1}\right)\right) .
\end{aligned}
$$


International Journal of Engineering Research and Technology. ISSN 0974-3154, Volume 13, Number 8 (2020), pp. 1854-1865

(C) International Research Publication House. http://www.irphouse.com

It is obvious that $a_{2}<0$. From Descartes' Rule of Signs [17], the conditions for positive $I^{*}$ of (10) is if $a_{0}<0$, then there are three positive $I^{*}$ or a positive $I^{*}$.

Next, we apply the Cardano formula [18] to get the analytical solution of (10), which formula was defined the variables as

$$
Q \equiv \frac{3 a_{1}-a_{2}^{2}}{9}, R \equiv \frac{9 a_{1} a_{2}-27 a_{0}-2 a_{2}^{3}}{54} .
$$

Then, the three roots can be obtained as follows. Define

$$
D \equiv Q^{3}+R^{2}, S \equiv(R+\sqrt{D})^{\frac{1}{3}}, T \equiv(R-\sqrt{D})^{\frac{1}{3}} .
$$

Then the roots of equation (10) are given by

$$
\begin{aligned}
& I_{1}^{*}=-\frac{1}{3} a_{2}+(S+T), I_{2}^{*}=-\frac{1}{3} a_{2}-(S+T)+\frac{1}{2} \mathrm{i} \sqrt{3}(S-T), \\
& I_{3}^{*}=-\frac{1}{3} a_{2}-(S+T)-\frac{1}{2} \mathrm{i} \sqrt{3}(S-T) .
\end{aligned}
$$

Equation (10) has a positive real solution when $D>0$ and $S+T>\frac{1}{3} a_{2}$. Then, $I^{*}$ in (10) is a positive real if the following conditions are satisfied

$4 a_{0} a_{2}^{3}-a_{1}^{2} a_{2}^{2}+4 a_{1}^{3}-162 a_{0} a_{1} a_{2}+27 a_{0}^{2}>0$ and $S+T>\frac{1}{3} a_{2}$,

where

$$
\begin{aligned}
& S=\left(\frac{9 a_{1} a_{2}-27 a_{0}-2 a_{2}^{3}}{54}+\sqrt{\left(\frac{3 a_{1}-a_{2}^{2}}{9}\right)^{3}+\left(\frac{9 a_{1} a_{2}-27 a_{0}-2 a_{2}^{3}}{54}\right)^{2}}\right)^{\frac{1}{3}}, \\
& T=\left(\frac{9 a_{1} a_{2}-27 a_{0}-2 a_{2}^{3}}{54}-\sqrt{\left(\frac{3 a_{1}-a_{2}^{2}}{9}\right)^{3}+\left(\frac{9 a_{1} a_{2}-27 a_{0}-2 a_{2}^{3}}{54}\right)^{2}}\right)^{\frac{1}{3}} .
\end{aligned}
$$

As noted above, $F^{*}=1 / b_{3}$ is also a possible equilibrium value. Repeating the steps given above, we find equilibrium points $E_{5}, E_{6}, E_{7}, E_{8}$. Hence, the equilibrium points are

$$
E_{5}=\left(\frac{s}{d_{1}}, 0,0, \frac{1}{b_{3}}\right), E_{6}=\left(\frac{s}{d_{1}}, 0, \frac{1}{b_{2}}, \frac{1}{b_{3}}\right), E_{7}=\left(I^{*}, T^{*}, 0, \frac{1}{b_{3}}\right)
$$

and $E_{8}=\left(I^{*}, T^{*}, N^{*}, \frac{1}{b_{3}}\right)$.

where $T^{*}=\frac{r_{1} r_{2} b_{1} b_{3} c_{3}-c_{3} c_{4} c_{5}-r_{1}^{2} r_{2} b_{1} b_{2}+r_{1} r_{2} b_{1} b_{2} c_{2} I^{*}}{r_{1} b_{1} b_{3}\left(c_{3} c_{4}-r_{1} r_{2} b_{1} b_{2}\right)}$

and $N^{*}=\frac{r_{1} b_{3} c_{4}-r_{1} r_{2} b_{1} b_{3}+c_{4} c_{5}-b_{3} c_{2} c_{4} I^{*}}{b_{3}\left(c_{3} c_{4}-r_{1} r_{2} b_{1} b_{2}\right)}$ with conditions for positive real are $c_{3} c_{4}>r_{1} r_{2} b_{1} b_{2}$ and $\frac{c_{3} c_{4} c_{5}+r_{1}^{2} r_{2} b_{1} b_{2}-r_{1} r_{2} b_{1} b_{3} c_{3}}{r_{1} r_{2} b_{1} b_{2} c_{2}}<$ $I^{*}<\frac{r_{1} b_{3} c_{4}+c_{4} c_{5}-r_{1} r_{2} b_{1} b_{3}}{b_{3} c_{2} c_{4}}$. The value of $I^{*}$ satisfies the cubic equation

$$
I^{* 3}+e_{2} I^{* 2}+e_{1} I^{*}+e_{0}=0
$$

Where

$$
\begin{gathered}
e_{2}=\frac{1}{r_{1} r_{2} b_{1} b_{2} b_{3} c_{2}}\left(c_{1}\left(r_{1} r_{2} b_{1} b_{3} c_{3}+c_{3} c_{4} c_{5}-r_{1}^{2} r_{2} b_{1} b_{2} b_{3}\right)-r_{1} b_{1} b_{3}\left(\rho-\alpha c_{1}-d_{1}\right)\left(c_{3} c_{4}-r_{1} r_{2} b_{1} b_{2}\right)\right), \\
e_{1}=\frac{c_{1}}{\left(c_{1} r_{1} r_{2} b_{1} b_{2} b_{3} c_{2}\right)^{2}}\left(d_{1} \alpha r_{1}^{2} b_{1}^{2} b_{3}^{2}\left(c_{3} c_{4}-r_{1} r_{2} b_{1} b_{2}\right)^{2}-s r_{1}^{2} r_{2} b_{1}^{2} b_{2} b_{3}^{2} c_{2}\left(c_{3} c_{4}-r_{1} r_{2} b_{1} b_{2}\right)\right. \\
\quad+c_{1}\left(r_{1}^{2} r_{2}^{2} b_{1}^{2} b_{3}^{2} c_{3}^{2}+c_{3}^{2} c_{4}^{2} c_{5}^{2}+r_{1}^{4} r_{2}^{2} b_{1}^{2} b_{2}^{2} b_{3}^{2}+2 r_{1} r_{2} b_{1} b_{3} c_{3}^{2} c_{4} c_{5}-2 r_{1}^{3} r_{2}^{2} b_{1}^{2} b_{2} b_{3}^{2}\right.
\end{gathered}
$$

$$
\begin{aligned}
& \left.-2 r_{1}^{2} r_{2} b_{1} b_{2} b_{3} c_{3} c_{4} c_{5}\right)-r_{1} b_{1} b_{3}\left(\rho-\alpha c_{1}-d_{1}\right)\left(c_{3} c_{4}-r_{1} r_{2} b_{1} b_{2}\right)\left(r_{1} r_{2} b_{1} b_{3} c_{3}+c_{3} c_{4} c_{5}\right. \\
& \left.\left.-r_{1}^{2} r_{2} b_{1} b_{2} b_{3}\right)\right) \\
e_{0}= & \frac{c_{1}}{\left(c_{1} r_{1} r_{2} b_{1} b_{2} b_{3} c_{2}\right)^{2}}\left(s r_{1} b_{1} b_{3}\left(c_{3} c_{4}-r_{1} r_{2} b_{1} b_{2}\right)\left(r_{1}^{2} r_{2} b_{1} b_{2} b_{3}-r_{1} r_{2} b_{1} b_{3} c_{3}-c_{3} c_{4} c_{5}\right)-s \alpha\right) .
\end{aligned}
$$

From Descartes' Rule of Signs, we can find the conditions for positive $I^{*}$ of (11) as follows

(1) If $e_{2}>0$ and $e_{0}<0$, then there is a positive $I^{*}$.

(2) If $e_{2}<0$ and $e_{0}<0$, then there are three positive $I^{*}$ or a positive $I^{*}$.

Using the Cardano solution of (11), the conditions for the value of $I^{*}$ to be a positive real number are

$$
4 e_{0} e_{2}^{3}-e_{1}^{2} e_{2}^{2}+4 e_{1}^{3}-162 e_{0} e_{1} e_{2}+27 e_{0}^{2}>0 \text { and } S+T>\frac{1}{3} e_{2} .
$$

where

$$
\begin{aligned}
& S=\left(\frac{9 e_{1} e_{2}-27 e_{0}-2 e_{2}^{3}}{54}+\sqrt{\left(\frac{3 e_{1}-e_{2}^{2}}{9}\right)^{3}+\left(\frac{9 e_{1} e_{2}-27 e_{0}-2 e_{2}^{3}}{54}\right)^{2}}\right)^{\frac{1}{3}}, \\
& T=\left(\frac{9 e_{1} e_{2}-27 e_{0}-2 e_{2}^{3}}{54}-\sqrt{\left(\frac{3 e_{1}-e_{2}^{2}}{9}\right)^{3}+\left(\frac{9 e_{1} e_{2}-27 e_{0}-2 e_{2}^{3}}{54}\right)^{2}}\right)^{\frac{1}{3}} .
\end{aligned}
$$

In summary, the equilibrium points $E_{1}, E_{2}, E_{5}$ and $E_{6}$, the tumor cell populations were zero. These states were therefore medically desirable tumor-free states. In the equilibrium points $E_{1}, E_{2}, E_{5}$ and $E_{6}$, the tumor cell populations were nonzero. These were therefore endemic equilibrium states.

\section{STABILITY OF THE MODEL}

In this section, we study the local stability of the model (1) about each equilibria by the linearzation method. Let $W(t)=(I(t), T(t), N(t), F(t))^{T}$, then the linearzed of (1) about equilibrium $\left(I^{*}, T^{*}, N^{*}, F^{*}\right)$ is given as follows

$$
\begin{aligned}
\dot{W}(t) & =\left[\begin{array}{cccc}
-d_{1} & 0 & 0 & 0 \\
-c_{2} T^{*} & w_{22} & -c_{3} T^{*} & c_{5} T^{*} \\
0 & -c_{4} N^{*} & r_{2}-2 r_{2} b_{2} N^{*}-c_{4} T^{*} & 0 \\
0 & 0 & 0 & r_{3}-2 r_{3} b_{3} F^{*}
\end{array}\right] W(t) \\
& +\left[\begin{array}{cccc}
\frac{\rho T^{*}}{\alpha+T^{*}}-c_{1} T^{*} & \frac{\alpha \rho I^{*}}{\left(\alpha+T^{*}\right)^{2}}-c_{1} I^{*} & 0 & 0 \\
0 & 0 & 0 & 0 \\
0 & 0 & 0 & 0 \\
0 & 0 & 0 & 0
\end{array}\right] W(t-\tau),
\end{aligned}
$$

where $w_{22}=r_{1}-2 r_{1} b_{1} T^{*}-c_{2} I^{*}-c_{3} N^{*}+c_{5} F^{*}$. 
International Journal of Engineering Research and Technology. ISSN 0974-3154, Volume 13, Number 8 (2020), pp. 1854-1865

(C) International Research Publication House. http://www.irphouse.com

From (12), It is obvious that the equilibrium $E_{1}, E_{2}, E_{3}, E_{4}$ and $E_{5}$ are unstable for $\tau \geq 0$. Next, we show the local stability of the equilibrium $E_{6}$ for $\tau \geq 0$ as the following theorem

Theorem 2 If $s c_{2}>d_{1}\left(r_{1} b_{2} b_{3}+b_{2} c_{5}-b_{3} c_{3}\right)$, then $E_{6}$ of the model (1) is locally asymptotically stable for $\tau \geq 0$.

Proof: At $E_{6}$, the characteristics equation of the model (1) is given by

$$
\left|\begin{array}{cccc}
-d_{1}-\lambda & \left(\frac{\rho s}{d_{1} \alpha}-\frac{c_{1} s}{d_{1}}\right) e^{-\lambda \tau} & 0 & 0 \\
0 & r_{1}-\frac{c_{2} s}{d_{1}}-\frac{c_{3}}{b_{2}}+\frac{c_{5}}{b_{3}}-\lambda & 0 & 0 \\
0 & -\frac{c_{4}}{b_{2}} & -r_{2}-\lambda & 0 \\
0 & 0 & 0 & -r_{3}-\lambda
\end{array}\right|=0 .
$$

Hence, all eigenvalues of the characteristic of (13) are

$$
\lambda=-r_{2},-r_{3},-d_{1}, r_{1}-\frac{c_{2} s}{d_{1}}-\frac{c_{3}}{b_{2}}+\frac{c_{5}}{b_{3}} .
$$

Obviously, all roots associated with characteristics equation are negative if $s c_{2}>d_{1}\left(r_{1} b_{2} b_{3}+b_{2} c_{5}-b_{3} c_{3}\right)$.

Moreover, we can show that the global stability of the tumor free equilibrium point with zero time delay by using a Lyapunov function

Theorem 3 The tumor free equilibrium $E_{6}$ is globally asymptotically stable in positively invariant set $K$ with zero time delay if the condition

$$
s r_{1} c_{2} b_{1} b_{3}^{2}>\left(r_{1} b_{3}+c_{5}\right)\left(c_{1} r_{1} b_{3}+c_{1} c_{5}+r_{1} b_{1} b_{3} d_{1}\right)
$$

is fulfilled.

Proof: We introduce a Lyapunov function [19] of the form $V(T)=T$, which is positive-definite and continuously differentiable for all positive bounded values of $T$, i.e., $V(0)=0$ and $V(T)>0, \forall T>0$. Hence, the time derivative of the Lyapunov function $V$ satisfies

$$
\begin{aligned}
\dot{V} & =\dot{T}, \\
& =r_{1} T\left(1-b_{1} T\right)-c_{2} I T-c_{3} T N+c_{5} T F, \\
& \leq T\left(r_{1}-r_{1} b_{1} T_{\text {min }}-c_{2} I_{\text {min }}-c_{3} N_{\text {min }}+c_{5} F_{\text {max }}\right) .
\end{aligned}
$$

If $r_{1}-c_{2} I_{\min }+c_{5} F_{\max }<0$ then, we will get $\dot{V} \leq 0$. From Theorem 1, we can simplify the condition as

$$
s r_{1} c_{2} b_{1} b_{3}^{2}>\left(r_{1} b_{3}+c_{5}\right)\left(c_{1} r_{1} b_{3}+c_{1} c_{5}+r_{1} b_{1} b_{3} d_{1}\right) \text {. }
$$

We should note that the Lyapunov conditions in (14) are a sufficient condition and are not necessary for global stability of the tumor free equilibrium $E_{6}$.

\section{EXISTENCE OF PERIODIC SOLUTION}

In this section, we focus on bifurcation behavior of $E_{8}$ of model (1) when $\tau \geq 0$. First, we determine local stability at $E_{8}$. From (12), the characteristic equation of the model (1) about equilibrium $E_{8}$ is given as following

$$
\left|\begin{array}{cccc}
-d_{1}+v_{11} e^{-\lambda \tau}-\lambda & v_{12} e^{-\lambda \tau} & 0 & 0 \\
-c_{2} T^{*} & u_{22}-\lambda & -c_{3} T^{*} & c_{5} T^{*} \\
0 & -c_{4} N^{*} & u_{33}-\lambda & 0 \\
0 & 0 & 0 & -r_{3}-\lambda
\end{array}\right|=0 \text {, }
$$

where

$u_{22}=r_{1}-2 r_{1} b_{1} T^{*}-c_{2} I^{*}-c_{3} N^{*}+\frac{c_{5}}{b_{3}}, u_{33}=r_{2}-2 r_{2} b_{2} N^{*}-c_{4} T^{*}$,

$v_{11}=\frac{\rho T^{*}}{\alpha+T^{*}}-c_{1} T^{*}$ and $v_{12}=\frac{\alpha \rho I^{*}}{\left(\alpha+T^{*}\right)^{2}}-c_{1} I^{*}$.

which gives

$$
\left(-r_{3}-\lambda\right)\left(\lambda^{3}+n_{3} \lambda^{2}+n_{2} \lambda+n_{1}+\left(n_{6} \lambda^{2}+n_{5} \lambda+n_{4}\right) e^{-\lambda \tau}\right)=0,
$$

where

$n_{1}=-d_{1}\left(c_{3} c_{4} T^{*} N^{*}-u_{22} u_{33}\right), n_{2}=-d_{1} u_{22}-d_{1} u_{33}+u_{22} u_{33}-c_{3} c_{4} T^{*} N^{*}$, $n_{3}=d_{1}-u_{22}-u_{33}, n_{4}=c_{3} c_{4} T^{*} N^{*} v_{11}-c_{2} T^{*} u_{33} v_{12}-u_{22} u_{33} v_{11}$, $n_{5}=u_{22} v_{11}+u_{33} v_{11}+c_{2} T^{*} v_{12}$ and $n_{6}=-v_{11}$.

From (15), we find that one of characteristic is $\lambda=-r_{3}<0$. Hence, if $\tau=0$ then, equation (15) will become

$$
\lambda^{3}+n_{11} \lambda^{2}+n_{12} \lambda+n_{13}=0
$$

where $n_{11}=d_{1}-\left(u_{22}+u_{33}+v_{11}\right)$,

$$
\begin{aligned}
& n_{12}=\left(v_{11}-d_{1}\right)\left(u_{22}+u_{33}\right)+c_{2} T^{*} v_{12}+u_{22} u_{33}-c_{3} c_{4} T^{*} N^{*}, \\
& n_{13}=\left(d_{1}+v_{11}\right) c_{3} c_{4} T^{*} N^{*}+d_{1} u_{22} u_{33} .
\end{aligned}
$$

From [12] the solution of a cubic equation was published by Gerolano Cardano. Hence, the solutions of the equation (16) in $\lambda$ are given by

$$
\lambda_{1}=-\frac{1}{3} n_{11}+(S+T)
$$

$$
\begin{aligned}
& \lambda_{2}=-\frac{1}{3} n_{11}-(S+T)+\frac{1}{2} \mathrm{i} \sqrt{3}(S-T), \\
& \lambda_{3}=-\frac{1}{3} n_{11}-(S+T)-\frac{1}{2} \mathrm{i} \sqrt{3}(S-T) .
\end{aligned}
$$

From the Cardano formula, we found that if $D>0$ then, $\lambda_{1}$ is real number, $\lambda_{2}$ and $\lambda_{3}$ are a pair of conjugate complex roots. 
International Journal of Engineering Research and Technology. ISSN 0974-3154, Volume 13, Number 8 (2020), pp. 1854-1865

(C) International Research Publication House. http://www.irphouse.com

If $D \leq 0$ then, $\lambda_{1}, \lambda_{2}$ and $\lambda_{3}$ are real numbers. Hence, the stability of the equilibrium $E_{8}$ of the model (1) when $\tau=0$ can be stated in the following theorem.

Theorem 4 If $|S+T|<\frac{1}{3} n_{11}$ and $|(S-T)|<\frac{4 \sqrt{3}}{9} n_{11}$, then the stability of $E_{8}$ of the model (1) with $\tau=0$ is asymptotically stable where

$$
\begin{aligned}
& n_{11}=d_{1}-\left(u_{22}+u_{33}+v_{11}\right), \\
& S=\left(\frac{9 n_{12} n_{11}-27 n_{13}-2 n_{11}^{3}}{54}+\sqrt{\left(\frac{3 n_{12}-n_{11}^{2}}{9}\right)^{3}+\left(\frac{9 n_{12} n_{11}-27 n_{13}-2 n_{11}^{3}}{54}\right)^{2}}\right)^{\frac{1}{3}}, \\
& T=\left(\frac{9 n_{12} n_{11}-27 n_{13}-2 n_{11}^{3}}{54}-\sqrt{\left(\frac{3 n_{12}-n_{11}^{2}}{9}\right)^{3}+\left(\frac{9 n_{12} n_{11}-27 n_{13}-2 n_{11}^{3}}{54}\right)^{2}}\right)^{\frac{1}{3}} .
\end{aligned}
$$

Proof: From the Cardano formula, we can stated as follow.

In case $D>0$, if $|S+T|<\frac{1}{3} n_{11}$, we find that $\lambda_{1}$ is negative real number and real parts of $\lambda_{2}$ and $\lambda_{3}$ are negative. Hence, the equilibrium $E_{8}$ is asymptotically stable. Otherwise, if $|S+T|>\frac{1}{3} n_{11}$ then, $\lambda_{1}$ is positive real number. Hence, the equilibrium $E_{8}$ is unstable.

In case $D \leq 0$, if $|S+T|<\frac{1}{3} n_{11}$, we find that $\lambda_{1}$ is negative real number. If $D=0$ and $|S+T|<\frac{1}{3} n_{11}$, we get $S=T=R^{\frac{1}{3}}$ and then, $\lambda_{2}=-\frac{1}{3} n_{11}-2 R^{\frac{1}{3}}$ and $\lambda_{3}=-\frac{1}{3} n_{11}-2 R^{\frac{1}{3}}$. Hence, $\lambda_{1}, \lambda_{2}$ and $\lambda_{3}$ are negative real number.

If $D<0$, we have

$$
S=(R+\sqrt{|D|})^{\frac{1}{3}} \text { and } T=(R-\sqrt{|D|})^{\frac{1}{3}}
$$

Hence, we get the root of $S$ and $T$ are

$$
S_{k}=r^{\frac{1}{3}}\left(\cos \left(\frac{\theta}{3}+\frac{2 k \pi}{3}\right)+\mathrm{i} \sin \left(\frac{\theta}{3}+\frac{2 k \pi}{3}\right)\right)
$$

and

$$
T_{k}=r^{\frac{1}{3}}\left(\cos \left(\frac{\theta}{3}+\frac{2 k \pi}{3}\right)-\mathrm{i} \sin \left(\frac{\theta}{3}+\frac{2 k \pi}{3}\right)\right) ; k=0,1,2 .
$$

where

$$
r=\sqrt{Q^{3}+2 R^{2}} \text { and } \theta=\tan ^{-1}\left(\frac{\sqrt{|D|}}{R}\right)
$$

Hence,

$$
S+T=2 r^{\frac{1}{3}} \cos \left(\frac{\theta}{3}+\frac{2 k \pi}{3}\right) \text { and } S-T=2 r^{\frac{1}{3}} \sin \left(\frac{\theta}{3}+\frac{2 k \pi}{3}\right) i
$$

From Gerolano Cardano formula, the solutions of cubic equation are

$$
\begin{aligned}
& \lambda_{1}=-\frac{1}{3} n_{11}+(S+T), \\
& \lambda_{2}=-\frac{1}{3} n_{11}-2 r^{\frac{1}{3}} \cos \left(\frac{\theta}{3}+\frac{2 k \pi}{3}\right)+\sqrt{3} r^{\frac{1}{3}} \sin \left(\frac{\theta}{3}+\frac{2 k \pi}{3}\right), \\
& \lambda_{3}=-\frac{1}{3} n_{11}-2 r^{\frac{1}{3}} \cos \left(\frac{\theta}{3}+\frac{2 k \pi}{3}\right)-\sqrt{3} r^{\frac{1}{3}} \sin \left(\frac{\theta}{3}+\frac{2 k \pi}{3}\right) .
\end{aligned}
$$

We find that $\lambda_{1}$ is negative real number if $|S+T|<\frac{1}{3} n_{11}$. For $\lambda_{2}$ if $|S+T|<\frac{1}{3} n_{11}$ then

$$
0<\frac{1}{3} n_{11}+2 r^{\frac{1}{3}} \cos \left(\frac{\theta}{3}+\frac{2 k \pi}{3}\right)<\frac{2}{3} n_{11} .
$$

Let $\lambda_{2}<0$ then,

$$
\sqrt{3} r^{\frac{1}{3}} \sin \left(\frac{\theta}{3}+\frac{2 k \pi}{3}\right)<\frac{1}{3} n_{11}+2 r^{\frac{1}{3}} \cos \left(\frac{\theta}{3}+\frac{2 k \pi}{3}\right) .
$$

From (17) and (18), the condition of $\lambda_{2}$ is negative real number as follows

$$
\sqrt{3} r^{\frac{1}{3}} \sin \left(\frac{\theta}{3}+\frac{2 k \pi}{3}\right)<\frac{2}{3} n_{11}
$$

For $\lambda_{3}:$ if $|S+T|<\frac{1}{3} n_{11}$ then

$$
-\frac{2}{3} n_{11}<-\frac{1}{3} n_{11}-2 r^{\frac{1}{3}} \cos \left(\frac{\theta}{3}+\frac{2 k \pi}{3}\right)<0 .
$$

Let $\lambda_{3}<0$ then,

$$
-\frac{1}{3} n_{11}-2 r^{\frac{1}{3}} \cos \left(\frac{\theta}{3}+\frac{2 k \pi}{3}\right)<\sqrt{3} r^{\frac{1}{3}} \sin \left(\frac{\theta}{3}+\frac{2 k \pi}{3}\right) \text {. }
$$

From (20) and (21), the condition of $\lambda_{3}$ is negative real number as follows

$$
-\frac{2}{3} n_{11}<\sqrt{3} r^{\frac{1}{3}} \sin \left(\frac{\theta}{3}+\frac{2 k \pi}{3}\right) .
$$

Hence, from ( 19) and (22), we find that $\lambda_{2}$ and $\lambda_{3}$ are negative real number which the condition as follows

$$
|(S-T)|<\frac{4 \sqrt{3}}{9} n_{11} .
$$


International Journal of Engineering Research and Technology. ISSN 0974-3154, Volume 13, Number 8 (2020), pp. 1854-1865

(C) International Research Publication House. http://www.irphouse.com

Hence, the equilibrium $E_{8}$ is asymptotically stable. Otherwise, if $|S+T|>\frac{1}{3} n_{11}$ then, $\lambda_{1}$ is positive real number. Hence, the equilibrium $E_{8}$ is unstable.

Next, we focus on bifurcation behavior of $E_{8}$ of model (1) when $\tau>0$. First, we restate the characteristic equation of (15) as follow:

$$
\lambda^{3}+n_{3} \lambda^{2}+n_{2} \lambda+n_{1}+\left(n_{6} \lambda^{2}+n_{5} \lambda+n_{4}\right) e^{-\lambda \tau}=0,
$$

where

$$
\begin{aligned}
& n_{1}=-d_{1}\left(c_{3} c_{4} T^{*} N^{*}-u_{22} u_{33}\right), n_{2}=-d_{1} u_{22}-d_{1} u_{33}+u_{22} u_{33}-c_{3} c_{3} \\
& n_{3}=d_{1}-u_{22}-u_{33}, n_{4}=c_{3} c_{4} T^{*} N^{*} v_{11}-c_{2} T^{*} u_{33} v_{12}-u_{22} u_{33} v_{11}, \\
& n_{5}=u_{22} v_{11}+u_{33} v_{11}+c_{2} T^{*} v_{12} \text { and } n_{6}=-v_{11} .
\end{aligned}
$$

Suppose that $\omega \in \mathcal{R}^{+}$and let $\lambda=i \omega$ be roots of (24). Then, we get

$$
\begin{aligned}
\omega^{6}+\left(n_{3}^{2}-2 n_{2}-n_{6}^{2}\right) \omega^{4}+\left(n_{2}^{2}-2 n_{1} n_{3}+2 n_{4} n_{6}-n_{5}^{2}\right) \omega^{2} \\
+\left(n_{1}^{2}-n_{4}^{2}\right)=0 .
\end{aligned}
$$

Let $\omega^{2}=z>0$, so the equation (25) can be reduced as follows

$$
z^{3}+r_{1} z^{2}+r_{2} z+r_{3}=0
$$

where $\quad r_{1}=n_{3}^{2}-2 n_{2}-n_{6}^{2}, r_{2}=n_{2}^{2}-2 n_{1} n_{3}+2 n_{4} n_{6}-n_{5}^{2} \quad$ and $r_{3}=n_{1}^{2}-n_{4}^{2}$. From [20] the solution of the cubic equation was published by Gerolano Cardano. Hence, the solutions of the equation (26) in $z$ are given by

$$
\begin{aligned}
& z_{1}=-\frac{1}{3} r_{1}+(S+T), z_{2}=-\frac{1}{3} r_{1}-(S+T)+\frac{1}{2} \mathrm{i} \sqrt{3}(S-T), \\
& z_{3}=-\frac{1}{3} r_{1}-(S+T)-\frac{1}{2} \mathrm{i} \sqrt{3}(S-T) .
\end{aligned}
$$

The solution of the equation (26) be a positive solution when $D>0$ and $S+T>\frac{1}{3} r_{1}$. From the conditions we can find at least one of $\omega_{k} ; k=1,2,3$ is a positive solution. Hence, a positive solution of (25) is given as follows

$$
\omega=\sqrt{S+T-\frac{1}{3} r_{1}}
$$

Finally, we will find $\tau_{k}$ in the form as follows

$$
\tau_{k}=\frac{1}{\omega} \cos ^{-1}\left(\frac{\left(n_{5}-n_{3} n_{6}\right) \omega^{4}+\left(n_{1} n_{6}+n_{3} n_{4}-n_{2} n_{5}\right) \omega^{2}-n_{1} n_{4}}{\left(n_{5} \omega\right)^{2}+\left(n_{6} \omega^{2}-n_{4}\right)^{2}}+2 k \pi\right),
$$

where $k=0,1,2, \ldots$
Condition (27) represents the bifurcation points of the model (1). We present the theorem for the bifurcation points $\tau_{0}$ is Hopf bifurcation points in the following theorem.

Theorem 5 Let $\lambda(\tau)=\alpha(\tau)+i \omega(\tau)$ be a root of (16) $\tau=\tau_{0}$ satisfying $\alpha\left(\tau_{0}\right)=0$ and $\omega\left(\tau_{0}\right)=\omega$ where $\omega \in R^{+}$and $\tau_{0}$ is the smallest positive root of (27). If all parameters of (1) meet the conditions

$$
0<\omega^{2}<\frac{2 n_{4} n_{6}-n_{5}^{2}}{2 n_{6}^{2}}, 2 n_{1} n_{3}>n_{2}^{2} \text { and } 2 n_{2}<n_{3}^{2},
$$

then $\operatorname{Re}\left(\left.\frac{d \lambda}{d \tau}\right|_{\tau=\tau_{0}}\right)>0$ and hence $\tau=\tau_{0}$ is the Hopf $N^{*}$, bifurcation point of (1).

Proof: To prove additional conditions in Theorem 8, consider the characteristic equation in the form as follows

$$
\lambda^{3}+n_{3} \lambda^{2}+n_{2} \lambda+n_{1}+\left(n_{6} \lambda^{2}+n_{5} \lambda+n_{4}\right) e^{-\lambda \tau}=0 .
$$

Let $\lambda$ be a function of $\tau$, i.e. $\lambda=\lambda(\tau)$. Differentiate both sides of (28) with respect to $\tau$, and let $\lambda\left(\tau_{0}\right)=i \omega_{0}$, we can show that

$$
\begin{aligned}
\left.\left(\frac{d \lambda}{d \tau}\right)^{-1}\right|_{\tau=\tau_{0}} & =\frac{\left[\left(n_{2}-3 \omega^{2}\right)+\mathrm{i} 2 n_{3} \omega\right]\left[\left(n_{2} \omega^{2}-\omega^{4}\right)-\mathrm{i}\left(n_{3} \omega^{3}-n_{1} \omega\right)\right]}{\left(n_{2} \omega^{2}-\omega^{4}\right)^{2}+\left(n_{3} \omega^{3}-n_{1} \omega\right)^{2}} \\
& +\mathrm{i} \frac{\tau}{\omega}+\frac{\left[n_{5}+\mathrm{i} 2 n_{6} \omega\right]\left[-n_{5} \omega^{2}-\mathrm{i}\left(n_{4} \omega-n_{6} \omega^{3}\right)\right]}{n_{5}^{2} \omega^{4}+\left(n_{4} \omega-n_{6} \omega^{3}\right)^{2}} .
\end{aligned}
$$

Consider only the real part of (29). It follows that

$$
\begin{aligned}
\operatorname{Re}\left(\left.\left(\frac{d \lambda}{d \tau}\right)^{-1}\right|_{\tau=\tau_{0}}\right)= & \frac{\left(3 \omega^{4}+2 n_{3}^{2} \omega^{2}+n_{2}^{2}\right)-\left(4 n_{2} \omega^{2}+2 n_{1} n_{3}\right)}{\left(n_{2} \omega-\omega^{3}\right)^{2}+\left(n_{3} \omega^{2}-n_{1}\right)^{2}} \\
& +\frac{2 n_{4} n_{6}-\left(2 n_{6}^{2} \omega^{2}+n_{5}^{2}\right)}{n_{5}^{2} \omega^{2}+\left(n_{4}-n_{6} \omega^{2}\right)^{2}}
\end{aligned}
$$

We can see that $\operatorname{Re}\left(\left.\left(\frac{d \lambda}{d \tau}\right)^{-1}\right|_{\tau=\tau_{0}}\right)>0$ when $3 \omega^{4}+2 n_{3}^{2} \omega^{2}$ $+n_{2}^{2}>4 n_{2} \omega^{2}+2 n_{1} n_{3}$ and $2 n_{4} n_{6}>2 n_{6}^{2} \omega^{2}+n_{5}^{2}$. Hence, the condition can be simplified as

$$
\frac{\sqrt{\left(n_{3}^{4}+4 n_{2}^{2}+18 n_{1} n_{3}\right)-\left(9 n_{2}^{2}+4 n_{2} n_{3}^{2}\right)}+2 n_{2}-n_{3}^{2}}{3}<\omega^{2}<\frac{2 n_{4} n_{6}-n_{5}^{2}}{2 n_{6}^{2}} .
$$

If $2 n_{1} n_{3}>n_{2}^{2}$ and $2 n_{2}<n_{3}^{2}$ then, the condition (30) becomes

$$
0<\omega^{2}<\frac{2 n_{4} n_{6}-n_{5}^{2}}{2 n_{6}^{2}} .
$$

Thus $\tau_{0}$ is the Hopf bifurcation point.

Hence, all works above shows that the hypotheses for Hopf bifurcation are satisfied at $\tau=\tau_{0}$ with the condition (31). These lead us to state the following theorem.

Theorem 6 The conditions for stability of model (1) at $E_{8}$, we have the following.

(1) If $\tau<\tau_{0}$, then the equilibrium point $E_{8}$ is asymptotically stable. 
International Journal of Engineering Research and Technology. ISSN 0974-3154, Volume 13, Number 8 (2020), pp. 1854-1865

(C) International Research Publication House. http://www.irphouse.com

(2) If $\tau>\tau_{0}$, then the equilibrium point $E_{8}$ is unstable.

(3) If $\tau=\tau_{0}$, then Hopf bifurcation occurs when $2 n_{1} n_{3}>n_{2}^{2}$, $0<\omega_{0}^{2}<\frac{2 n_{4} n_{6}-n_{5}^{2}}{2 n_{6}^{2}}, 2 n_{2}<n_{3}^{2}$ and $\tau_{0}$ is the Hopf bifurcation point.

\section{NUMERICAL SIMULAITON RESULTS}

In this section, we implement mathematical programs of Maple software package to simulate numerical results for local and global stability, necessary conditions of behavior of bifurcation and effecting some parameters for tumor growth. The parameter values in Table 2 and four types of initial conditions $(t=0)$ can apply for our numerical simulations of the model (1).

Table 2: The value of parameters for the tumor-obesity model

\begin{tabular}{|c|c|c|c|}
\hline Parameters & $\begin{array}{c}\text { Values } \\
\text { used }\end{array}$ & Unit & Reference \\
\hline$s$ & 0.33 & Density. $m L^{-1} \cdot d a y^{-1}$ & [10] \\
\hline$\rho$ & 0.01 & $d a y^{-1}$ & {$[10]$} \\
\hline$\alpha$ & 0.3 & Density.mL & [10] \\
\hline$d_{1}$ & 0.2 & $d a y^{-1}$ & [10] \\
\hline$r_{1}$ & 0.5 & $d a y^{-1}$ & Estimated \\
\hline$r_{2}$ & 1 & $d a y^{-1}$ & [10] \\
\hline$r_{3}$ & 0.3025 & $d a y^{-1}$ & Estimated \\
\hline$b_{1}$ & 1 & Density. $m L$ & [10] \\
\hline$b_{2}$ & 0.5 & Density.mL & Estimated \\
\hline$b_{3}$ & 0.4 & Density.mL & Estimated \\
\hline$c_{1}$ & 1 & Density. $m L^{-1} \cdot d a y^{-1}$ & [10] \\
\hline$c_{2}$ & 1 & Density $m L^{-1} \cdot d a y^{-1}$ & Estimated \\
\hline$c_{3}$ & 1 & Density $m L^{-1} \cdot d a y^{-1}$ & {$[10]$} \\
\hline$c_{4}$ & 1 & Density $m L^{-1} \cdot d a y^{-1}$ & [10] \\
\hline$c_{5}$ & 0.1 & Density. $m L^{-1} \cdot$ day $^{-1}$ & Estimated \\
\hline
\end{tabular}

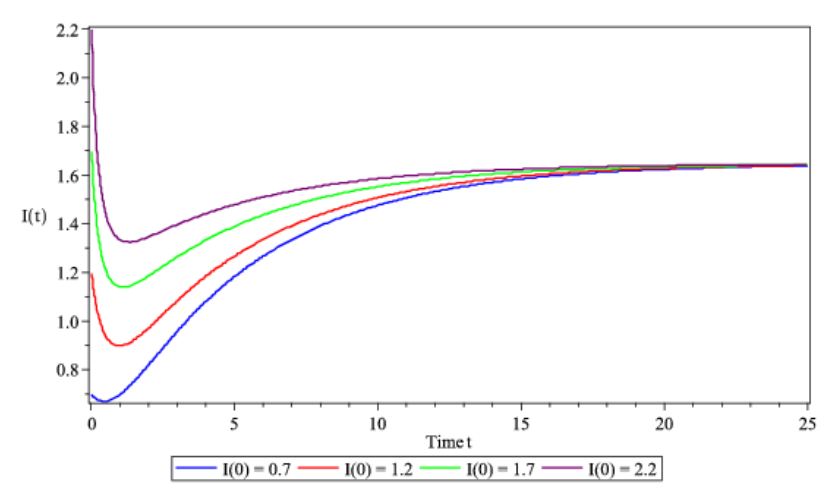

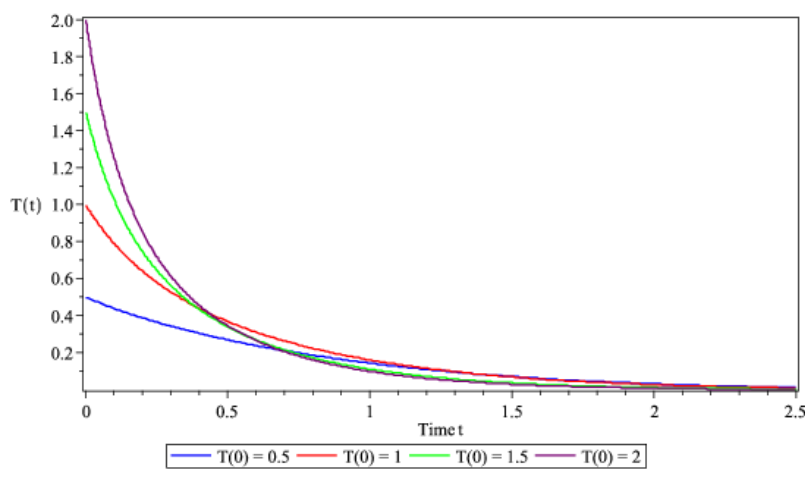
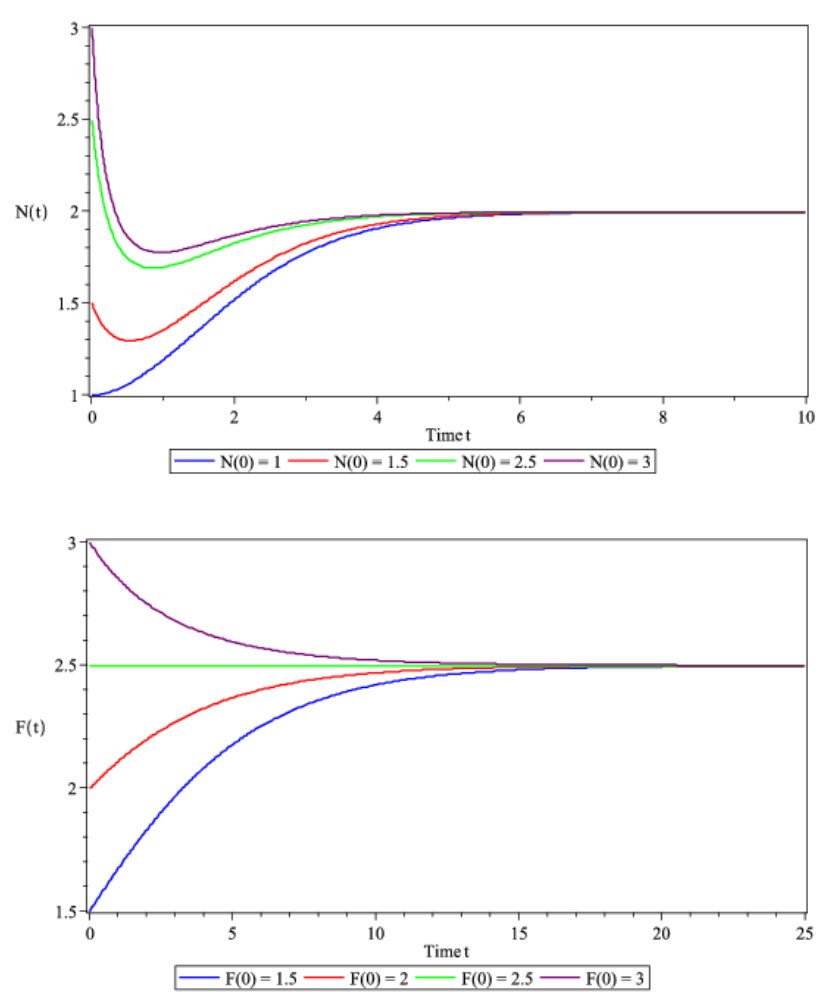

Figure 1: The numerical simulation of Immune cells, Tumor cells, Host cells and Fat cells with different initial conditions

Graphs in Figure 1 with different initial conditions shows that all numerical solutions for the host population classes converge to $E_{6}=(1.65,0,2,2.5)$. Those results agree with the theoretical results provided in Theorem 2, which show that the equilibrium point $E_{6}$ is asymptotically stable.

Next, we provide the numerical simulations of the model (1) with the parameter values in Table 3 and the initial conditions

$$
I(0)=0.52, T(0)=0.94, N(0)=1.3, F(0)=1.1,
$$

which gives the endemic equilibrium point is

$$
E_{8}=(0.505541473,0.9206153173,1.284981766,1)
$$

and the Hopf bifurcation point $\tau_{0}$ in (27) is given by $\tau_{0} \approx 8.167$. 
International Journal of Engineering Research and Technology. ISSN 0974-3154, Volume 13, Number 8 (2020), pp. 1854-1865

(C) International Research Publication House. http://www.irphouse.com

Table 3: The value of parameters for the tumor-obesity model

\begin{tabular}{|c|c|c|c|}
\hline Parameters & $\begin{array}{c}\text { Values } \\
\text { used }\end{array}$ & Unit & Reference \\
\hline$s$ & 0.33 & Density. $m L^{-1} \cdot d a y^{-1}$ & {$[10]$} \\
\hline$\rho$ & 0.01 & $d a y^{-1}$ & {$[10]$} \\
\hline$\alpha$ & 0.3 & Density.mL & {$[10]$} \\
\hline$d_{1}$ & 0.2 & $d a y^{-1}$ & {$[10]$} \\
\hline$r_{1}$ & 1.5 & $d a y^{-1}$ & {$[10]$} \\
\hline$r_{2}$ & 1 & $d a y^{-1}$ & [10] \\
\hline$r_{3}$ & 0.3 & $d a y^{-1}$ & Estimated \\
\hline$b_{1}$ & 0.8 & Density. $m L$ & Estimated \\
\hline$b_{2}$ & 0.42 & Density.mL & Estimated \\
\hline$b_{3}$ & 1 & $\begin{array}{l}\text { Densily. } m L \\
\text { Density. } m L\end{array}$ & Estimated \\
\hline$c_{1}$ & 0.5 & Density. $m L^{-1} \cdot d a y^{-1}$ & Estimated \\
\hline$c_{2}$ & 0.5 & Density. $m L^{-1} \cdot d a y^{-1}$ & Estimated \\
\hline$c_{3}$ & 0.5 & Density $m L^{-1} \cdot d a y^{-1}$ & Estimated \\
\hline$c_{4}$ & 0.5 & Density. $m L^{-1} \cdot$ day $^{-1}$ & Estimated \\
\hline$c_{5}$ & 0.5 & Density. $m L^{-1} \cdot$ day $^{-1}$ & Estimated \\
\hline
\end{tabular}

Based on Theorem 6, all numerical solutions for the host population classes converge to $E_{8}$ for all time delays $\tau$, if $0 \leq \tau<\tau_{0} \approx 8.167$. Graphs of numerical solutions in Figure 2 are decreasingly oscillated to the equilibrium point which gives asymptotically stability, when $\tau=7.5<\tau_{0} \approx 8.167$. The Hopf bifurcation phenomena can be occurred when a bifurcation point, $\tau$, is sufficiently large and across $\tau_{0} \approx 8.167$. For example, $\tau=8.18>\tau_{0} \approx 8.167$, the solutions of immune cells, tumor cells and normal cells are widely oscillated about the equilibrium $E_{8}$ in Figure 3. Moreover, if obese people are tumors, the density of fat cells are rapidly decreasing, while the density of tumor cells are constantly oscillating and changing any time $t$.

Finally, we are interested in two parameters: $r_{3}$ (the growth rate for the density of fat cells) and $b_{3}$ (the inverse of the carrying capacity for the density of fat) in order to showing the effective parameters for decreasing the level of density for tumor cells in the model (1). Choosing three decreasing $r_{3}=1.2,1.1,1.0$ and three increasing $b_{3}=0.98,0.99,1.00$ and the value of a time delay $\tau_{0} \in[7.5,8.16]$. The level of density of tumor cells in the model (1) are decay and changing its behavior from limit cycle to asymptotically stable at $\tau=7.49$ in Figure 4 In the other hand, selecting $\tau=8.2$, the level of density of tumor cell in the model (1) is widely oscillating and changing its behavior from uncontrolled to limit cycle to a periodic level in Figure 5.
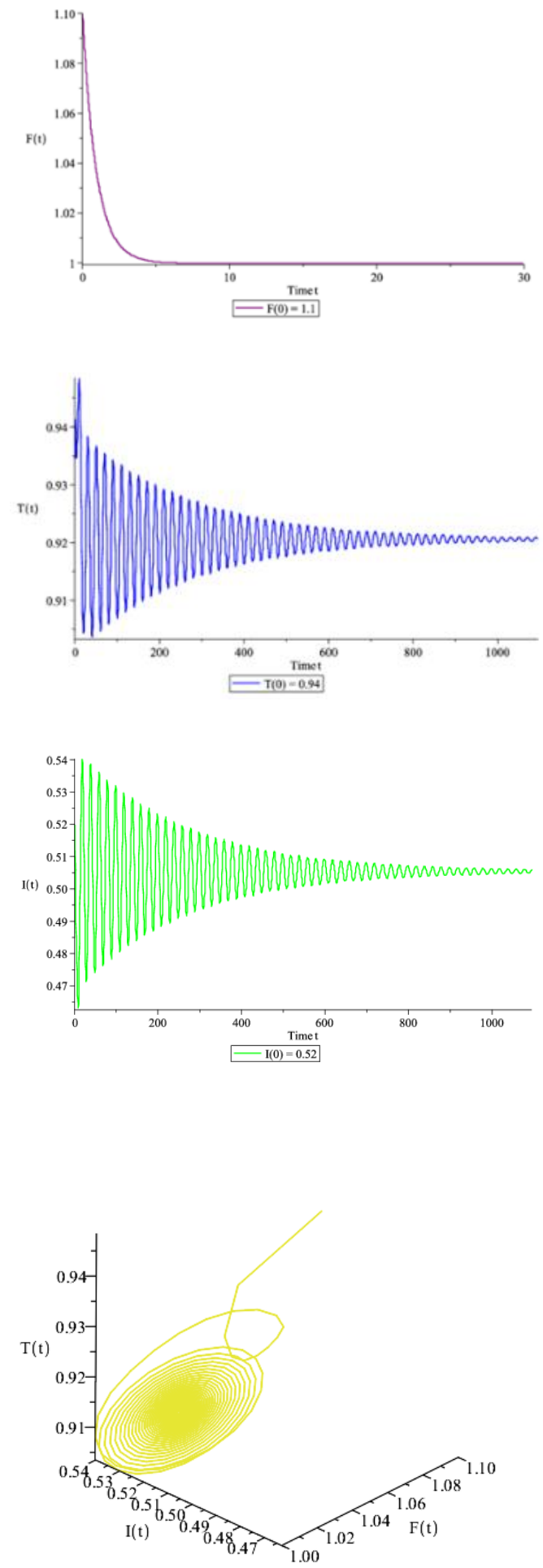

Figure 2: The numerical simulation of Immune cells, Tumor cells, Normal cells and Fat cells when $\tau=7.5$ 
International Journal of Engineering Research and Technology. ISSN 0974-3154, Volume 13, Number 8 (2020), pp. 1854-1865 (C) International Research Publication House. http://www.irphouse.com
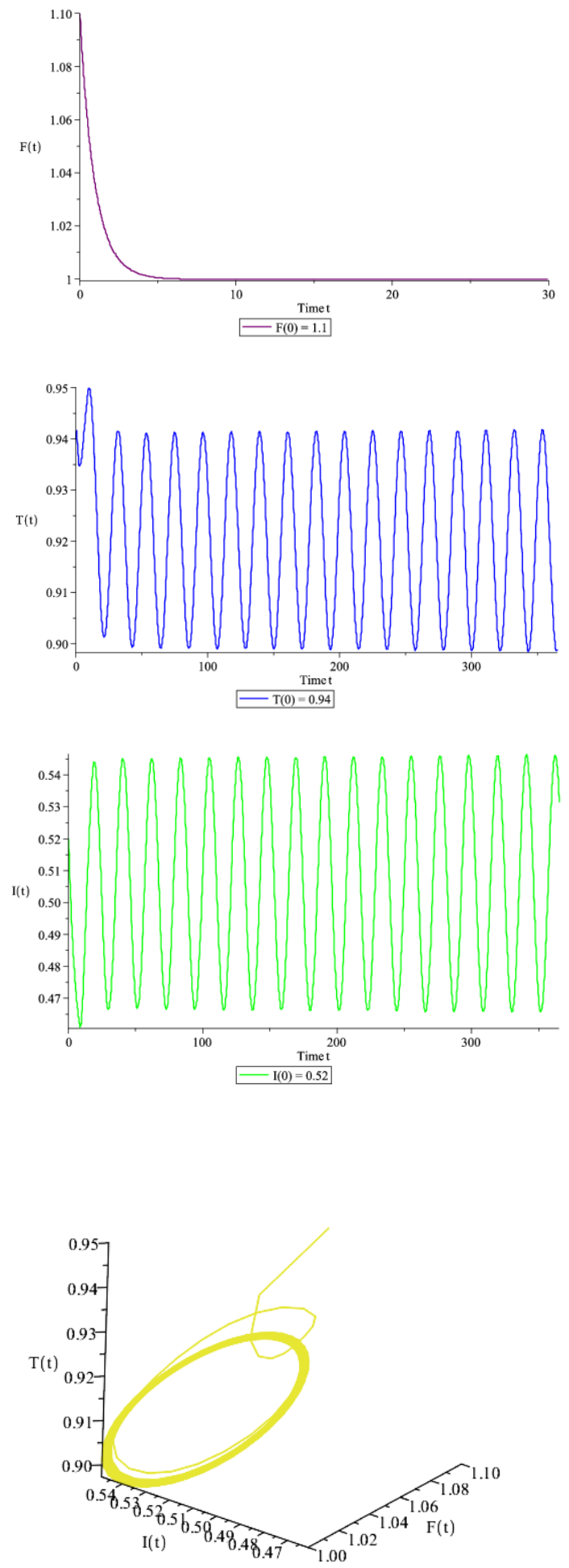

Figure 3: The numerical simulation of Immune cells, Tumor cells, Normal cells and Fat cells when $\tau=8.18$
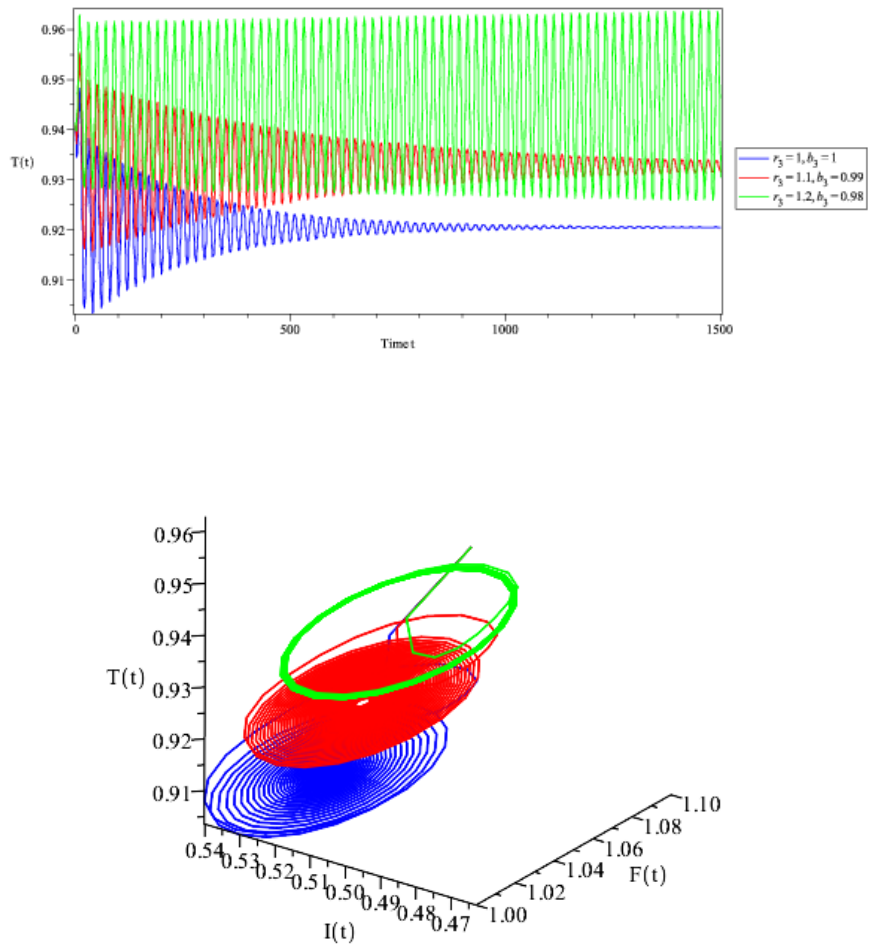

Figure 4: Time plots of Tumor cells and the numerical simulation of Immune cells, Tumor cells and Fat cells with different values of $r_{3}$ and $b_{3}$ at $\tau=7.49$
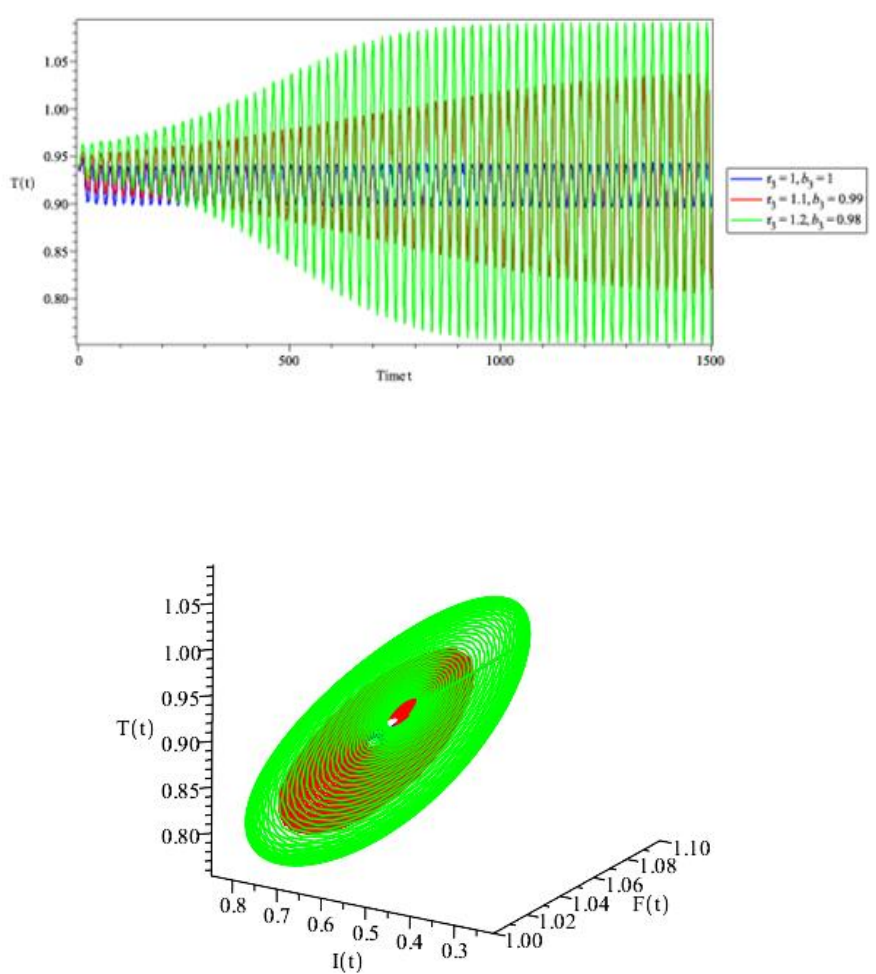

Figure 5: Time plots of Tumor cells and the numerical simulation of Immune cells, Tumor cells and Fat cells with different values of $r_{3}$ and $b_{3}$ at $\tau=8.2$ 


\section{CONCLUSION}

In this paper, we presented an analysis of the obesity and tumor models both with and without time delay. We first used the LCIS method (Theorem 1) to find the bounds of a domain containing all compact invariant sets of models without time delay for positive values of model parameters. The generalized Gronwall Lemma applied for finding the bounds of $I(t)$ of model with time delay which is uniformly bounded. For stability of equilibrium points, we found that equilibrium points $E_{1}, E_{2}, E_{3}, E_{4}$ and $E_{5}$ of the models both with/without a time delay are always unstable. Next, the statement and proof of the conditions for local stability of $E_{6}$ is given in Theorem 2. Also, the conditions for the global stability of $E_{6}$ with zero-time delay are stated and proved in Theorem 3. Therefore, we analyzed the stability of the endemic equilibrium points $E_{8}$, and proved that the equilibrium by linearization was stable for the model without time delay in Theorem 4 . For the time-delay model, we derived the necessary conditions for the existence of a Hopf bifurcation point (Theorem 5). In the numerical simulations, we used biologically reasonable values of parameters to test our analytical results. We found that the numerical simulations converged to the equilibrium point $E_{6}$ for choices of parameter values satisfying the conditions in Theorem 2-3. The numerical simulations also showed convergence to $E_{8}$ for time delays $\tau$ less than the Hopf bifurcation point $\tau_{0}$ and limit cycle behavior for $\tau>\tau_{0}$ (Theorem 6). Also, the effect of some parameters on the dynamic of the model (1) are studied for the value of $r_{3}$ (the growth rate for the density of fat cells) decreases and the value of $b_{3}$ (the inverse of the carrying capacity for the density of fat) increases. It can be concluded that the obesity and time delay affect the growth of tumors. The obese people are likely to increase the density of the tumor and the obesity is a health problem of the modern world.

\section{ACKNOWLEDGEMENT}

This research is supported by the Centre of Excellence in Mathematics, the Commission on Higher Education, the Department of Mathematics, Faculty of Applied Science, King Mongkut's University of Technology North Bangkok and Kasem Bundit University, Thailand

\section{REFERENCES}

[1] S. Friberg and S. Mattson, "On the growth rates of human malignant tumors: implications for medical decision making," Journal of surgical oncology, vol. 65, no. 4, pp. 284-297, 1997.

[2] F.J. Santonja, A. Morales, R.J. Villanueva, and J.C. Cortes, "Analysing the effect of public health campaigns on reducing excess weight: A modelling approach for the Spanish autonomous region of the community of Valencia," Evaluation and program planning, vol. 35, no. 1, pp. 34-39, 2012.
[3] C. Oh and M. MA, "Optimal intervention strategies for the spread of obesity," Journal of Applied Mathematics, 2015.

[4] D. Aldila, N. Rarasati, N. Nuraini, and E. Soewono, "Optimal control problem of treatment for obesity in a closed population," International Journal of Mathematics and Mathematical Sciences, 2014.

[5] D. Laudisio, G. Muscogiuri, L. Barrea, S. Savastano, and A. Colao, "Obesity and breast cancer in premenopausal women: Current evidence and future perspectives," European Journal of Obstetrics and Gynaecology and Reproductive Biology, vol. 230, pp. 217-221, 2018.

[6] C.A.A.Rojas, M.T.Alvarez-Banuelos, J.Morales Romero, H.Suarez-Diaz, J. C. Hernandez-Fonseca, and G. Contreras-Alarcon, "Breast cancer: Metastasis, molecular subtypes, and overweight and obesity in Veracruz, Mexico," Clinical breast cancer, vol. 19, no. 1, pp. 166-171, 2019.

[7] H. Rubin, "Promotion and selection by serum growth factors drive field cancerization, which is anticipated in vivo by type 2 diabetes and obesity," Proceedings of the National Academy of Sciences, vol. 110, no. 34, pp. 13927-13931, 2013.

[8] S. D. Hursting, "Minireview: the year in obesity and cancer," Molecular endocrinology, vol. 26, no. 12, pp. 1961-1966, 2012.

[9] M. Schwab, Encyclopaedia of cancer. Springer Science and Business Media, 2008.

[10] R. A. Ku-Carrillo, S. E. Delgadillo, and B. ChenCharpentier, "A mathematical model for the effect of obesity on cancer growth and on the immune system response," Applied Mathematical Modelling, vol. 40, no. 7, pp. 4908-4920, 2016.

[11] J. Jo, O. Gavrilova, S. Pack, W. Jou, S. Mullen, A. E. Sumner, S. W. Cushman, and V. Periwal, "Hypertrophy and/or hyperplasia: dynamics of adipose tissue growth," PLoS computational biology, vol. 5, no. 3, 2009.

[12] D. Okwan-Duodu, G. E. Umpierrez, O. W. Brawley, and R. Diaz, "Obesity-driven inflammation and cancer risk: role of myeloid derived suppressor cells and alternately activated macrophages," American journal of cancer research, vol. 3, no. 1, 2013.

[13] M. Villasana and A. Radunskaya, "A delay differential equation model for tumor growth," Journal of Mathematical Biology, vol. 47, no. 3, pp. 270-294, 2003.

[14] F. A. Rihan, D. Abdelrahman, F. Al-Maskari, F. Ibrahim, and M. A. Abdeen, "Delay differential model for tumour-immune response with chemoimmunotherapy and optimal control," Computational and math- ematical methods in medicine, 2014.

[15] P. A. Valle, K. E. Starkov, and L. N. Coria, "Global stability and tumor clearance conditions for a cancer 
International Journal of Engineering Research and Technology. ISSN 0974-3154, Volume 13, Number 8 (2020), pp. 1854-1865

(C) International Research Publication House. http://www.irphouse.com

chemotherapy system," Communications in Nonlinear Science and Numerical Simulation, vol. 40, pp. 206$215,2016$.

[16] A. Halanay and A. Halanay, Differential equations: Stability, oscillations, time lags, vol. 6. Academic press New York, 1966.

[17] D. J. Grabiner, "Descartes' rule of signs: Another construction," The American Mathematical Monthly, vol. 106, no. 9, pp. 854-856, 1999.

[18] E. Weisstein, "Np-hard problem, mathworld a wolfram web resource," 2005.

[19] P. Liu and X. Liu, "Dynamics of a tumor-immune model considering targeted chemotherapy," Chaos, Solutions and Fractals, vol. 98, pp. 7- 13, 2017.

[20] E. W. Weisstein and C. Problem, "From mathworld a wolfram web re- source http://mathworld.wolfram. com," Mean Curvature. html, 2005.

[21] Sarud U, Sanoe K, Ekkachai K. The effect of obesity and cancer stem cells in tumor model with time delay, 9(4), COMPUSOFT, An International Journal of Advanced Computer Technology. PP. 3633-3641. 